\title{
Beobachtungen kleiner Planeten
}

am 8-inch Refraktor der Breslauer Sternwarte.

\begin{tabular}{l|l|l|l|l|l|l|l|l|}
\hline Datum & M.Z. Breslau & $\Delta \alpha$ & $\Delta \delta$ & Vgl. & $\alpha$ app. & $\log p . \Delta \mid \delta$ app. & $\log p . \Delta \mid$ Red.ad l.app. & $*$ \\
\hline
\end{tabular}

r 907

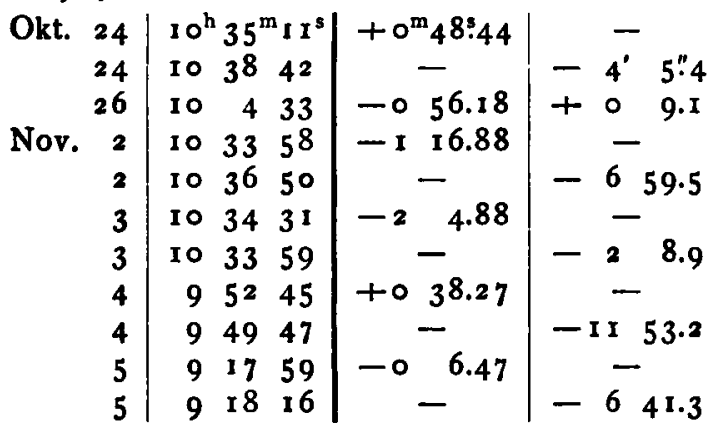

1908

Jan. I 5

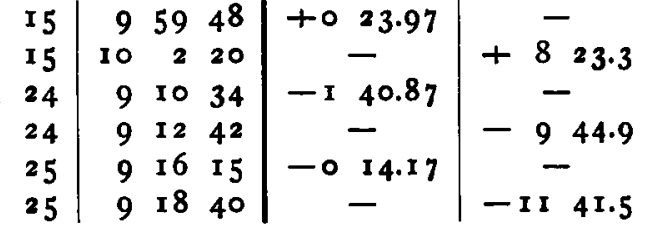

(47I) Papagena.

\begin{tabular}{|c|c|c|c|c|c|c|c|c|c|}
\hline $\begin{array}{l}8 \\
8\end{array}$ & $\begin{array}{c}1^{\mathrm{h}} 4^{2^{\mathrm{m}}} 4^{6: 3} \cdot 34 \\
-\end{array}$ & $\begin{array}{c}8.9^{8} 7 \mathrm{n} \\
-\end{array}$ & $-14^{\circ}$ & $\overline{1}^{\prime}$ & $51 " 1$ & $0 . \overline{899}$ & $\begin{array}{c}+2.62 \\
-\end{array}$ & $\begin{array}{r}-\overline{13} \cdot 5 \\
+13\end{array}$ & $\mathbf{I}$ \\
\hline$p, 8 s$ & $141 \quad 1.73$ & $9.122 n$ & -13 & 57 & 36.9 & 0.896 & +2.63 & +13.2 & I \\
\hline $\begin{array}{l}8 \\
8\end{array}$ & I $35{ }^{1.2 \mathrm{I}}$ & $\begin{array}{c}8.45^{2} \mathrm{n} \\
-\end{array}$ & -13 & $\overline{33}$ & 56.0 & $0 . \overline{899}$ & $\begin{array}{c}+2.64 \\
-\end{array}$ & $\begin{array}{c}-\overline{1} \\
+\mathrm{I2.5}\end{array}$ & 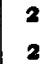 \\
\hline 4 & I $34 \quad 13.22$ & $8.29 I_{n}$ & & - & & - & +2.65 & - & \\
\hline 4 & - & - & -13 & 29 & $5 \cdot 5$ & 0.899 & 一 & +12.4 & \\
\hline 7 & I 3327.69 & $8.906_{n}$ & & - & & - & +2.64 & - & \\
\hline 8 & 一 & - & $-I_{3}$ & 24 & 25.2 & 0.897 & - & +12.3 & \\
\hline 4 & 13242.95 & $9.108 n_{n}$ & & - & & - & +2.64 & 一 & \\
\hline 6 & - & $\pi$ & -13 & 10 & 13.4 & 0.894 & - & +12.2 & \\
\hline
\end{tabular}

(532) Herculina.

Mittlere Örter der Vergleichsterne.

\begin{tabular}{|c|c|c|c|}
\hline * & $\alpha 1907.0$ & $\delta 1907.0$ & Autorität \\
\hline $\mathbf{I}$ & $I^{h} 4 I^{m} 55^{s} \cdot 28$ & $-13^{\circ} 57^{\prime} 59^{\prime \prime} .2$ & $\mathrm{San}_{1}$ I 16 \\
\hline 2 & I $36 \quad 15.45$ & $\begin{array}{lll}-1327 & 9.0\end{array}$ & 108 \\
\hline 3 & I 3246.78 & - I 3 I 244.3 & 1103 \\
\hline
\end{tabular}

Breslau, I 908 Juni 4 .

\begin{tabular}{|c|c|c|c|}
\hline$*$ & $\alpha 1908.0$ & $\delta 1908.0$ & Autorität \\
\hline 4 & $7^{\mathrm{h}} I 7^{\mathrm{m}} 5^{6} \cdot 5^{\mathrm{s}}$ & $+23^{\circ} 7^{\prime} 24^{\prime \prime} .5$ & AG B \\
\hline 5 & 7 I I 12.49 & +244157.8 & 280 \\
\hline 6 & 7849.77 & +24528.6 & 284 \\
\hline
\end{tabular}

H. Wolff.

\section{Une variable nouvelle 14.1908 Draconis.}

Sur des plaques dues à M. S. Blažko, Mme. L. Ceraski a trouvé ce 6 juin une variable nouvelle qui est $\mathrm{BD}+49^{\circ} 2820\left(9^{\mathrm{m}} \cdot 5\right)$.

Ses coordonnées d'après la $\mathrm{BD}$ sont les suivantes:

\subsection{Draconis.}

$$
\begin{array}{rlrl}
1855.0 & \alpha= & =8^{\mathrm{h}} 30^{\mathrm{m}} \text { 1 s.o } & \delta=+49^{\circ} 12.7 \\
1900.0 & =18 \text { 3 } 10.1 & =+4914.7
\end{array}
$$

A en juger d'après les 25 photographies obtenues entre r900 et r907, son éclat varie de $9^{\text {m }}$ d $<12^{m}$. M. Blažko trouve que la période de cette étoile est apparemment longue, peut-être de quelques mois.

Moscou, le I 4 juin 1908 .

Prof. W. Ceraski.

\section{Inhalt:}

Zu Nr. 4259. E. D. Roe. Observations and measures of double stars. 169. $-M$. Luizet. Sur quelques étoiles variables nouvelles. I 71. - M. Luizet. Sur l'etoile variable RW Dragon. 175. - F. Fr. Schroeter. Einige Berichtigungen und Ergänzungen zu Burnhams General Catalogue of double stars. 179. - A. Abetti. Rettifica. 181. $-H$. Wolff. Beobachtungen kleiner Planeten. 183. $-W$. Ceraski. Une variable nouvelle 14.1908 Draconis. 183. 